www.jmscr.igmpublication.org

Impact Factor (SJIF): 6.379

Index Copernicus Value: 71.58

ISSN (e)-2347-176x ISSN (p) 2455-0450

crossref DOI: https://dx.doi.org/10.18535/jmscr/v6i2.163

Journal Of Medical Science And Clinical Research

IGM Publication

An Official Publication of IGM Publication

\title{
A Prospective Observational study to Identify the Incidence and Predictors of difficult and Impossible Mask Ventilation
}

\author{
Authors \\ Dr Mallika Balakrishnan, Dr Mathana V, Dr Bhagyalakshmi Ramesh, \\ Dr Jagathnath Krishna \\ Regional Cancer Centre Trivandrum, India
}

\begin{abstract}
Background: Mask Ventilation is the most fundamental skill of airway management and it is vital for the conduct of general anaesthesia ${ }^{1}$. Difficulties with airway management in relation to general anesthesia have been a challenge for the anesthesiologist since the birth of anesthesia. Considerable improvements have been made and general anesthesia is now regarded as a safe procedure. However, difficult airway situations still occur and it causes increased risk of morbidity and mortality especially when not anticipated ${ }^{2}$.In this study we intend to analyses the incidence of Difficult and Impossible Mask Ventilation (DMV) (IMV) and the factors associated with it.

Objective: To evaluate the preoperative specified clinical airway assessment parameters on prediction of $D M V$ and IMV.

Subjects and Methods: This study was a prospective observational study done in our institute among patients who were posted for elective surgery under general anesthesia.

Results: Two hundred patients who were mask ventilated during induction of general anesthesia were included for the study. Age >55yrs, Body mass index(BMI) of 30kg/m2 or greater, History of Diabetes $>5 y \mathrm{rs}$ duration, snoring, sternomental distance $(S M D)<12 \mathrm{~cm}$, Edentulous status, Modified Mallampatti classification III or IV, Thyromental distance grade II \& III, Upper Lip Bite Test (ULBT)class II \& III were identified as significant predictors for DMV by univariate analysis.

Conclusion: In our study population, Difficult Mask Ventilation was found in $11 \%$ of the patients. Independent risk factors for DMV include body mass index $>30 \mathrm{~kg} / \mathrm{m} 2$, thick obese neck, SMD $12 \mathrm{~cm}$ and lack of teeth. The DMV prediction may lead to a better preparedness for the management of difficult airway and also potentially decreasing the morbidity \& mortality resulting from hypoxia or anoxia associated with failed ventilation.
\end{abstract}

Keywords: Difficult Ventilation; Airway; Predictors; Mask ventilation.

\section{Introduction}

Good predictive index can alter and stratify the treatment for the individual patient and potentially improve the outcome. Prediction of difficult airway remains a pivotal challenge in anaesthesia and it is highly prioritized among anesthesia personnel so as to identify patients at risk of airway management difficulties ${ }^{3}$.

Difficult airway is defined as the clinical situation in which a conventionally trained anaesthesiologist experiences difficulty with face mask ventilation of the upper airway, difficulty with tracheal intubation, or both. ${ }^{4}$ 
Although there is an extensive body of literature addressing predictive factors for difficult laryngoscopy and grading its view, literature that focuses on Mask Ventilation are limited. Bag Mask Ventilation commonly precedes the establishment of a secure airway by endotracheal intubation during induction of GA. Successful identification of physical features that are suggestive of difficult mask ventilation will direct planning toward safe airway management ${ }^{5}$. Failure to access an airway during emergency or elective surgeries can result in hypoxic brain injury. Prediction of difficult mask ventilation (DMV) is therefore of vital importance ${ }^{6}$.

Mask ventilation is considered difficult when there is 1) Inability for the unassisted anaesthesiologist to maintain oxygen saturation of at least $92 \%$ using $100 \%$ oxygen and positive pressure ventilation, 2) Significant gas flow leak around the face mask, 3) necessity to increase the gas flow to greater than $15 \mathrm{~L} / \mathrm{min}$ and the need to use the oxygen flush valve more than twice, 4) Absence of perceptible chest movements 5) necessity to perform two-handed MV, and 6) requirement of change of operator ${ }^{3}$.

In 2003, the American Society of Anesthesiologists Task Force modified the definition of Impossible Mask Ventilation as the inability to provide adequate ventilation with a mask as evidenced by the absence or the inadequacy of breath sounds, chest movement, Spiro metric measures of exhaled gas flow, end tidal carbon dioxide (EtCO2) as well as inadequate oxygen saturation $(\mathrm{SpO} 2)$, cyanosis, the auscultatory signs of severe obstruction, clinical evidence of gastric insufflation and hemodynamic changes associated with hypoxemia or hypercarbia. ${ }^{7}$

Impossible mask ventilation is denoted by absence of end-tidal carbon dioxide measurement and lack of perceptible chest wall movement during positive pressure ventilation attempts despite airway adjuncts and additional personnel ${ }^{8}$.

No single predictor is solely capable of predicting difficult mask ventilation or difficult airway management. However, several studies indicate that by combining multiple predictors of difficult intubation the predictive value of the assessment increases ${ }^{9}$.

Research regarding DMV is limited although it is extremely important as it serves as a rescue technique for maintaining oxygenation in the scenario of Difficult or Impossible Endotracheal Intubation. In the face of DMV/IMV, critical hypoxemia may rapidly ensue and this emphasizes the need for proper identification of risk factors during preoperative assessment.

Accurate prediction of the difficult airway alters the potentially dangerous situation of an unanticipated airway to an anticipated difficult airway with ample time for adequate preparation with airway devices and adjuncts thereby reducing the possibility of airway catastrophes ${ }^{10}$.

Hence, this prospective observational study was done to find the incidence and predictive factors for the impossible and DMV in routine day to day conduct of General Anaesthesia.

Methods: After obtaining approval from the Institutional Review Board, subjects were selected from among those who were scheduled for general anesthesia after satisfying the inclusion criteria considered for the study. Details of the study were explained to the selected patients and their consent to participate in the study was sought.

For each subject preoperative history and physical examination was done. Details noted included a standard physical examination and airway assessment; physical features that may affect mask fit, patient history that may suggest difficult airway anatomy, pathology, and general condition of the patient and nature of the operation. Airway assessment included measurement of thyromental distance, sternomental distance, neck circumference, neck extension, mouth opening, modified Mallampati score and upper lip bite test. Standardized anaesthetic protocol was followed in all the patients. An appropriate anatomical face mask was selected. After establishing venous access and standard monitoring techniques, premedication with intravenous 
glycopyrrolate $0.2 \mathrm{mg}$, midazolam $(0.02 \mathrm{mg} / \mathrm{kg})$ and fentanyl (1-2 mcg/kg) was given. The patient was induced with intravenous lignocaine $2 \%$ (1 $\mathrm{mg} / \mathrm{kg}$ ) and propofol $(2 \mathrm{mg} / \mathrm{kg})$ and the lungs ventilated with $100 \%$ oxygen with help of a facemask. Mask ventilation assessment was done at the time of induction prior to the placement of a definitive airway by an experienced anesthetist with minimum two years of experience after postgraduation. Ease of Mask Ventilation was assessed in all subjects using mask ventilation scale At the end of the study, preoperative parameters like age of the patient, beard, dentition, cough, asthma, Diabetes Mellitus, snoring, sleep apnoea, body mass index, Mallampatti grade, abnormal cervical spine, thick obese neck, sternomental distance, palate, edentulous dentition, thyromental distance, mouth opening, upper lip bite test (18 parameters) were tabulated and correlated with intra-operative parameter (DMV, IMV) according to standard formulae to predict the difficult mask ventilation.

The sample size was calculated to be 200 based on number of variables to be analyzed. Data was entered in Microsoft Excel and Statistical Package for the Social Sciences (SPSS; Windows ver. 17.0, SPSS Inc., Chicago, IL) was used for statistical analysis. Quantitative data was described as Mean and Standard Deviation. Qualitative data was described by Frequency distribution. Univariate analysis was performed on the data having a grade 3 or grade $4 \mathrm{MV}$.

Statistical significance was tested using Pearson chi square test. $\mathrm{P}$ value less than 0.05 was considered significant. All variables found to be significant in the univariate analysis were entered into a multivariate logistic regression model to identify independent predictors of the measured outcome. Receiver operating characteristic curves were analyzed to assess the diagnostic value of the risk factor scale.

\section{Results}

Out of 200 patients, 147 patients had easy mask ventilation. We had difficulty in mask ventilating
53 patients. In 31 patients we were able to ventilate after improving mask seal and using airway adjuncts. In 22 patients we had to use twoprovider technique for adequate mask ventilation. Hence, 22 patients met the DMV criteria.

All the significant risk factors with $\mathrm{P}$ value $<0.05$ found by univariate analysis were included in multivariate analysis to identify independent predictors for difficult airway.

The independent predictor for DMV identified were $\mathrm{BMI}>30 \mathrm{~kg} / \mathrm{m} 2$ with a significant $\mathrm{P}$ value of 0.001 , sternomental distance $<12 \mathrm{~cm}$ with significant $\mathrm{P}$ value of $<0.001$, thick obese neck with significant $\mathrm{P}$ value of $<0.001$, edentulous status with $\mathrm{P}$ value of $<0.001$.

Hazard Ratio for BMI was 14.506 which means, BMI $>30 \mathrm{~kg} / \mathrm{m} 2$ have 14.5 times risk compared to BMI $<30 \mathrm{~kg} / \mathrm{m} 2$ for DMV. Similarly for Sternomental Distance $<12 \mathrm{~cm}$ and Thick Obese neck, the risk was 5 times more. For variable dentition, edentulous status had 4.4 times more risk for DMV.

In addition, receiver operator-characteristic (ROC) curves were used to assess the discriminative ability of various factors to predict DMV. The area under the ROC curve represents the probability that a randomly chosen patient with DMV is correctly ranked for a given risk factor with greater suspicion than a randomly chosen patient without DMV (i.e., the area under curve value of 0.5 means no apparent accuracy to predict DMV and the area under curve value of 1 indicates a perfect accuracy to predict DMV). ROC curve was analyzed to determine the best threshold that maximizes both sensitivity and specificity to obtain the best diagnostic accuracy. 
Table 1: Univariate Analysis-Significant Variables

\begin{tabular}{|c|c|c|c|c|c|}
\hline \multirow{2}{*}{\multicolumn{2}{|c|}{ Predictors }} & \multicolumn{2}{|c|}{ DMV } & \multirow{2}{*}{ Statistical Test } & \multirow{2}{*}{ P-value } \\
\hline & & Yes & $\mathrm{No}$ & & \\
\hline \multirow{3}{*}{ Age } & $<55$ & 8 & 109 & \multirow{3}{*}{ Fisher's Exact Test } & \multirow{3}{*}{0.009} \\
\hline & $55-80$ & 13 & 69 & & \\
\hline & $>80$ & 1 & 0 & & \\
\hline \multirow{3}{*}{ BMI } & $<30$ & 7 & 158 & \multirow{3}{*}{ Pearson Chi-Square } & \multirow{3}{*}{$<0.001$} \\
\hline & $30-40$ & 15 & 20 & & \\
\hline & $>40$ & 0 & 0 & & \\
\hline Diabetes & $>5$ yrs. & 8 & 31 & Pearson Chi-Square & 0.034 \\
\hline Snoring & yes & 9 & 10 & Pearson Chi-Square & $<0.001$ \\
\hline $\begin{array}{c}\text { Sternomental } \\
\text { distance }<12 \mathrm{~cm}\end{array}$ & yes & 7 & 4 & Fisher's Exact Test & $<0.001$ \\
\hline Thick obese neck & yes & 13 & 14 & Pearson Chi-Square & $<0.001$ \\
\hline \multirow{4}{*}{ MMT } & CLASS I & 0 & 24 & \multirow{4}{*}{ Fisher's Exact Test } & \multirow{4}{*}{$<0.001$} \\
\hline & CLASS II & 4 & 104 & & \\
\hline & CLASS III & 16 & 47 & & \\
\hline & CLASS IV & 2 & 3 & & \\
\hline \multirow{3}{*}{ TMD } & CLASS I & 0 & 30 & \multirow{3}{*}{ Pearson chi square test } & \multirow{3}{*}{$<0.001$} \\
\hline & CLASS II & 11 & 143 & & \\
\hline & CLASS III & 11 & 5 & & \\
\hline Edentulous & yes & 4 & 0 & Fisher's Exact Test & $<0.001$ \\
\hline \multirow{3}{*}{ ULBT } & CLASS I & 8 & 126 & \multirow{3}{*}{ Fisher's Exact Test } & \multirow{3}{*}{$<0.001$} \\
\hline & CLASS II & 11 & 48 & & \\
\hline & CLASS III & 2 & 0 & & \\
\hline
\end{tabular}

Graph 1: Univariate Analysis-Variables

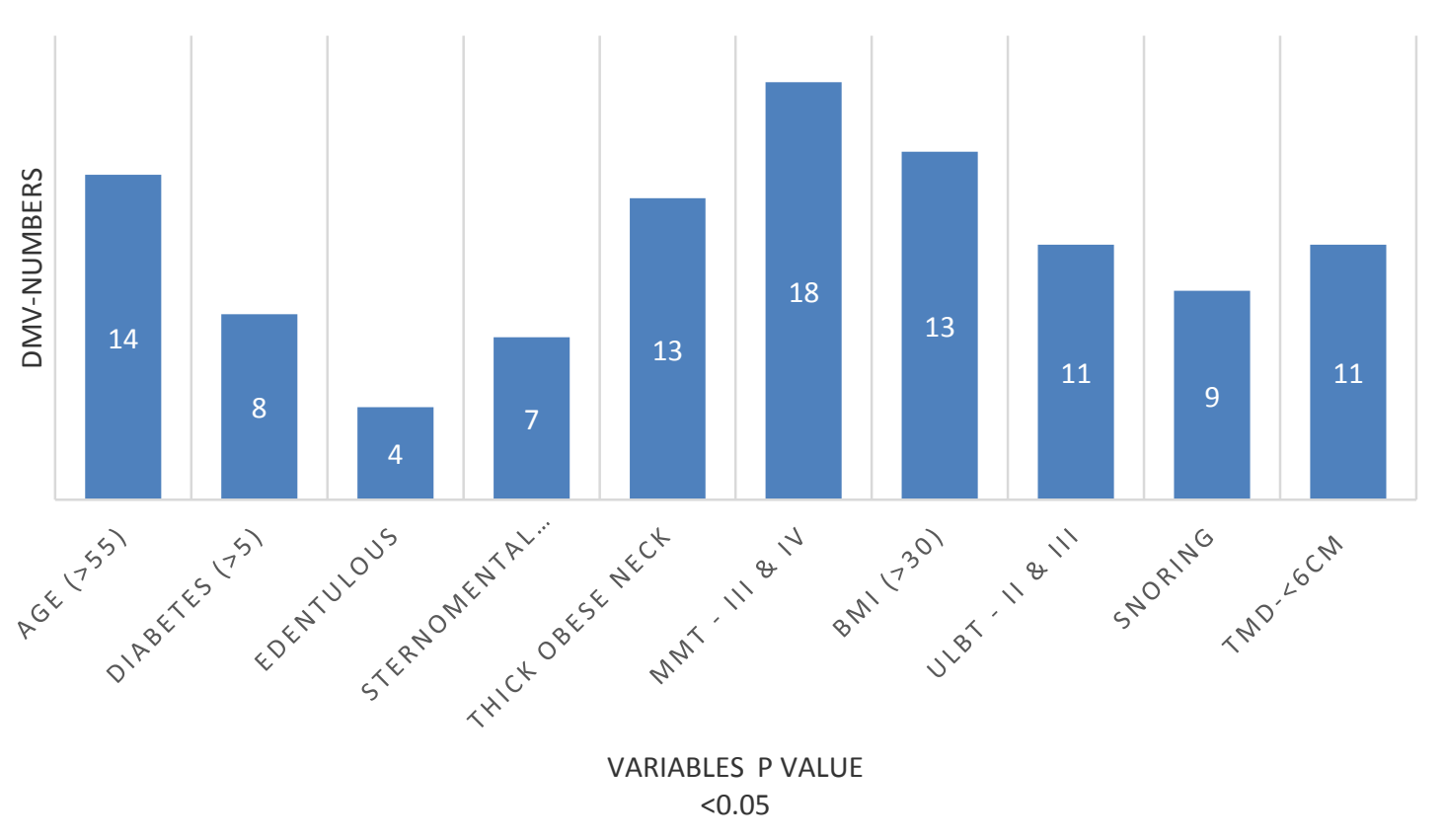


Table 2: Predictive power of independent risk factors of Difficult Mask Ventilation

\begin{tabular}{|l|c|c|c|c|}
\hline Variables & Sensitivity & Specificity & PPV & NPV \\
\hline BMI & 42.86 & 95.76 & 68.18 & 88.76 \\
\hline Sternomental Distance $<12 \mathrm{~cm}$ & 63.64 & 92.06 & 31.82 & 97.75 \\
\hline Thick obese neck & 48.15 & 94.80 & 59.09 & 92.13 \\
\hline Edentulous & 14.71 & 0.00 & 55.56 & 0.00 \\
\hline
\end{tabular}

\section{Multivariate Analysis}

Statistical Method: Multivariate Logistic Regression

Table 3: Cox multivariate analysis for the response variable DMV

\begin{tabular}{|l|c|c|c|c|}
\hline \multirow{2}{*}{ Variables } & \multirow{2}{*}{ P-Value } & \multirow{2}{*}{ Hazard ratio (HR) } & \multicolumn{2}{|c|}{ 95\% C.I. for HR } \\
\cline { 4 - 5 } & & & Lower & Upper \\
\hline BMI & 0.002 & 14.506 & 2.660 & 79.093 \\
\hline Sternomental Distance $<12 \mathrm{~cm}$ & 0.039 & 5.723 & 1.096 & 29.888 \\
\hline Thick Obese Neck & 0.035 & 5.029 & 1.117 & 22.641 \\
\hline Edentulous & 0.078 & 4.4 & .848 & 22.832 \\
\hline
\end{tabular}

Table 4: Predicting Difficult Mask Ventilation (DMV)

\begin{tabular}{|l|c|c|}
\hline Number Of Criteria & specificity & Negative predictive value \\
\hline 1 & 95.76 & 88.76 \\
\hline 2 & 88.72 & 97.19 \\
\hline 3 & 88.78 & 97.75 \\
\hline
\end{tabular}

\section{Discussion}

Although literature dealing with difficult airway is abundant, defining, measuring, and securing the difficult airway remains an elusive task. Differences among providers and between patients and regional variability in techniques and local strategies have all contributed to the challenge of generating consensus. The definition of DMV varies from study to study, but has included clinically relevant gas leak, frequent use of the oxygen flush valve, lack of chest movement, and/or desaturation. ${ }^{11}$ The specific incidence of DMV is rare, varying from 0.07 to $15 \%$.

In our prospective observational study, we found that the incidence of DMV was $11 \%$ and IMV was $0 \%$. Out of 200 patients, 22 patients had Difficult Mask Ventilation. The risk factors identified for DMV by univariate analysis were age $>55$ [14 out of 22], Diabetes $>5$ yrs. [8 out of 22], Snoring [9 out of 22], Edentulous status [4 out of 22], Sternomental Distance $<12 \mathrm{~cm}$ [7 out of 22], thick obese neck [13 out of 22], Mallampatti grade [III/IV] [18 out of 22], BMI $>30 \mathrm{~kg} / \mathrm{m} 2$ [15 out of 22], Thyromental distance $<6 \mathrm{~cm}$ [11 out of 22], ULBT class $2 \& 3$ [13 out of 22].
In our study none of the patients had impossible mask ventilation, so predictors for impossible mask ventilation could not be analyzed. Occurrence of DMV was more frequent in our study $(11 \%)$, similar to the findings ofShah and Sundaram et al. (DMV-12.82\%). ${ }^{12}$

Incidence of DMV has been rarely assessed in studies related to the airway management, and no previous specific study regarding difficulty with mask ventilation alone has been conducted. This may partly explain the discrepancies between our study and previous studies. ${ }^{3}$

In previous studies incidence of difficult mask ventilation could have been underestimated because these studies were based on self-reporting of adverse events by anesthesiologists, and based on the definition of DMV of American Society of Anaesthesiologists practice guidelines for management of the difficult airway and hence were restricted to peripheral oxygen saturation of 90\% without associated clinical signs of DMV.

Kheterpal et al reported BMI of $30 \mathrm{~kg} / \mathrm{m} 2$ or greater, presence of a beard, Mallampati classification III or IV, age of 57 yrs. or older, severely limited mandibular protrusion, and a 
history of snoring as independent risk factors for DMV.

In our study the independent predictors for DMV identified was $\mathrm{BMI}>30 \mathrm{~kg} / \mathrm{m} 2$ with a significant $P$ value of 0.001 , Short Neck with significant $P$ value of $<0.001$, Thick Obese Neck with significant $\mathrm{P}$ value of $<0.001$ and Edentulous status with significant $P$ value of $<0.001$. Independent risk factor identified by the multivariate analysis did not correlate with the study of Langeronet al in which BMI > $26 \mathrm{~kg} / \mathrm{m} 2$, lack of teeth, beard and snoring were the independent risk factors for DMV.

Shah and Sundaram et $\mathrm{al}^{12}$ found that risk factors for difficult mask ventilation were snoring, Obstructive sleep apnoea, retrognathia, micrognathia, macroglossia, Edentulous status, Short thick neck, Mallampatti Grade [III/IV] Grade, and BMI $>26 \mathrm{~kg} / \mathrm{m} 2$.Our findings were similar to Shah and Sundaram et al except that snoring was found to be significant in univariate analysis but not in the multivariate analysis findings.

Leoni et al studied 309 consecutive obese patients $\&$ his study revealed Neck Circumference (Odds Ratio 1.17; $\mathrm{P}<0.0001)$, limited protruded mandible (1.99; $\mathrm{P}=0.046)$ and Mallampati test (OR 2.12; $\mathrm{P}=0.009$ ) as risk predictors for DMV which is comparable to the results of our study which was a $\mathrm{P}$ value of $<0.05$ in univariate analysis for Mallampatti grade III, IV, ULBT Class II, III.

Surprisingly OSA was not found to be an independent risk factor for DMV in our study even though obesity, thick obese neck and Sternomental Distance $<12 \mathrm{~cm}$ identified as independent risk factors for DMV similar to the study result of Davide Cattano et al. ${ }^{13}$

In our study edentulous patients had DMV, due to inadequate seal of the face mask and subsequent air leak around the mask causing difficulty in positive-pressure ventilation. Multivariate analysis for edentulous status showed only a marginal significance but analyzing accuracy of sensitivity and specificity by ROC curve of edentulous status revealed area under the curve to be 0.7 indicating high predictability of edentulous status for DMV.

Langeron et al studied 1504 patients and found that edentulous status was an independent risk factor for DMV with sensitivity 0.92 and specificity of 0.38 . Our study revealed a sensitivity of 14.71 with a positive predictive value of 55.56.

Varghese et al reported that sternomental distance compared to other tests like Mallampatti test and ULBT had higher specificity, Negative Predictive Value and Positive Predictive Value which makes it the single best test in predicting difficult intubation. Similarly in our study it was observed that patients with SMD $<12 \mathrm{~cm}$ had higher sensitivity, specificity compared to other tests which indicates its significance as an accurate predictor for DMV. The higher NPV resulting from using more variables is of distinct advantage to the anesthesiologist as it offers the option of performing a trial laryngoscopy after administration of muscle relaxants without fear of inadequate oxygenation with BMV. Our study results cannot be extrapolated to a pediatric population or high-risk populations for difficult intubation, such as ear/nose/throat, obstetric, or emergency patients.

The incidence of impossible ventilation was zero, and consequently this phenomenon could not be analyzed and its risk factors could not be identified. Patients with low pulmonary compliance or high airway resistance, associated with laryngospasm or bronchospasm, have an increased risk of DMV without any predicting factors of DMV, as described in our study.

The DMV /IMV is an indicator of a high risk for difficult airway and may lead to a better preparation and safety in difficult airway management, potentially decreasing the morbidity and mortality resulting from hypoxia or anoxia associated with failed ventilation ${ }^{12}$.In conclusion, our study reported DMV in $11 \%$ of cases and four criteria i.e., BMI $>30 \mathrm{~kg} / \mathrm{m} 2$, lack of teeth, sternomental distance $<12 \mathrm{~cm}$ and a thick obese neck were identified to be independent risk factors for DMV. 


\section{Recommendations}

Sternomental distance and neck circumference measurements should be included the preoperative assessment of the airway in order to predict difficult mask ventilation. Thyromental distance and upper lip bite test improves the predictive value of patients likely to have a DMV. Patients with a BMI $>30 \mathrm{~kg} / \mathrm{m}^{2}$, sternomental distance of $<12 \mathrm{~cm}$, with a thick obese neck and edentulous status, could be expected to have difficulties with mask ventilation. We must predict a difficult mask ventilation in order to anticipate a difficult airway to minimize any airway related morbidity with adequate time and preparation for airway management.

\section{Conclusions}

In our study population, Difficult Mask Ventilation was reported in $11 \%$ of the patients. None of the patients had impossible mask ventilation. The risk factors identified for DMV by univariate analysis were age $>55 \mathrm{yrs}$, Diabetes $>5$ yrs. duration, history of Snoring, Edentulous status, Sternomental Distance $<12 \mathrm{~cm}$, thick obese neck, Mallampatti grade [III/IV], BMI $>30 \mathrm{~kg} / \mathrm{m} 2$, Thyromental distance grade II \& III, ULBT class II \& III. Independent factors identified for DMV body mass index $>30 \mathrm{~kg} / \mathrm{m} 2$, thick obese neck, sternomental distance $<12 \mathrm{~cm}$ \&lack of teeth. In our study DMV was managed by two provider technique of mask ventilation. No single predictor is sufficiently valid in predicting difficult airway. When combining multiple predictors of difficult airway the predictive value of the assessment increases. The prediction of DMV may lead to a better anticipation of difficult airway management, potentially decreasing the morbidity and mortality resulting from hypoxia or anoxia associated with failed ventilation.

\section{References}

1. Kheterpal S, Han R, Tremper KK, Shanks A, Tait AR, O'Reilly M, et al. Incidence and predictors of difficult and impossible mask ventilation. Anesthesiology. 2006 Nov; 105(5):885-91.

2. Nørskov AK. Preoperative airway assessment - experience gained from a multicenter cluster randomized trial and the Danish Anaesthesia Database. Dan Med J. 2016 May; 63(5).

3. Langeron O, Masso E, Huraux C, Guggiari $\mathrm{M}$, Bianchi A, Coriat $\mathrm{P}$, et al. Prediction of difficult mask ventilation. Anesthesiology. 2000 May; 92(5):1229-36.

4. American Society of Anesthesiologists Task Force on Management of the Difficult Airway. Practice guidelines for management of the difficult airway: an updated report by the American Society of Anesthesiologists Task Force on Management of the Difficult Airway. Anesthesiology. 2003 May;98(5):1269-77.

5. Baker P. Assessment before Airway Management. AnesthesiolClin. 2015 Jun 1; 33(2):257-78.

6. HossamAtef. The Effect of Position on Mallampati Airway Assessment Test in the Prediction of Difficult Mask Ventilation. Med J Cairo UnivVol 81 No 2 Sept 1-6 2013.

7. Practice Guidelines for Management of the Difficult Airway an Updated Report by the American Society of Anesthesiologists Task Force on Management of the Difficult Airway. J Am SocAnesthesiol. 2003 May 1; 98(5):1269-77.

8. Kheterpal S, Healy D, Aziz MF, Shanks $\mathrm{AM}$, Freundlich RE, Linton $\mathrm{F}$, et al. Incidence, Predictors, and Outcome of Difficult Mask Ventilation Combined with Difficult Laryngoscopy: A Report from the Multicenter Perioperative Outcomes Group. Anesthesiology. 2013 Dec; 119(6):1360-9.

9. Shiga T, Wajima Z, Inoue T, Sakamoto A. Predicting difficult intubation in apparently normal patients: a metaanalysis of bedside screening test 
performance. Anesthesiology. 2005 Aug; 103(2):429-37.

10. Cook TM, MacDougall-Davis SR. Complications and failure of airway management. Br J Anaesth. 2012 Dec 1; 109(suppl 1):i68-85.

11. D.B. Glick et al. The Difficult Airway: An Atlas of Tools and Techniques for Clinical Management. Springer Science+ Business Media New York; 2013.

12. Shah PN, Sundaram V. Incidence and predictors of difficult mask ventilation and intubation. J Anaesthesiol Clin Pharmacol. 2012; 28(4):451-5.

13. Cattano D, Katsiampoura A, Corso RM, Killoran PV, Cai C, Hagberg CA. Predictive factors for difficult mask ventilation in the obese surgical population. F1000Research [Internet]. 2014 Oct 9 [cited 2016 Nov 5]; 3. Available from: http://www.ncbi.nlm.nih.gov/pmc/articles/ PMC4244762.

\author{
Abbreviations \\ Difficult Mask Ventilation (DMV) \\ Impossible Mask Ventilation(IMV) \\ Body mass index(BMI) \\ Sternomental distance (SMD) \\ Upper Lip Bite Test (ULBT) \\ End tidal carbon dioxide (EtCO2)
}

